\title{
DIFFERENT TYPES OF DIFFUSION IN LIQUIDS BY QUASI-ELASTIC NEUTRON SCATTERING
}

\author{
A. Ya. Dzyublik, V. I. Slisenko, A. A. Vasilkevich \\ Institute for Nuclear Research of the Ukrainian Academy of Sciences \\ 47 Nauky, Pr., Kyiv, 03028, Ukraine \\ (Received April 4, 2001; received in final form September 25, 2001)
}

\begin{abstract}
A general expression for the incoherent quasi-elastic neutron scattering (QENS) cross section by a liquid is derived, taking into account the continuous translational and rotational diffusion of the so-called Lagrange particles (associations of atoms or molecules) as well as jumps of hydrogen atoms inside these particles. Both types of continuous motion are considered, starting from the Langevin equations. Besides, we carried out a QENS experiment on spirits, whose results agree well with our theory.
\end{abstract}

Key words: neutron scattering, liquid, diffusion, Lagrange particle, Langevin equation, Brownian rotation.

PACS number(s): 61.25.-f

\section{INTRODUCTION}

For a long time there have been two opposite approaches to the dynamics of liquids. The diffusion of their atoms (molecules) is described either as a continuous process or as jumps between adjacent sites (see, e.g., [1]). The continuous diffusion is determined by the diffusion or Langevin equations. However, simple diffusion equation cannot reproduce numerical experimental data on quasi-elastic scattering (QENS) by liquids [2-20]. Namely, the broadening of the elastic neutron peak $\Delta \varepsilon$, caused by the diffusive motion in a liquid, shows typical curved dependence on the squared transferred wave vector $Q^{2}$ (in particular, this is illustrated by Figs. 2-4 given below). At the same time the diffusion equation predicts linear function $\Delta \varepsilon\left(Q^{2}\right)$. Langevin equation, being more exact than the diffusion one, gives the Van Hove selfcorrelation function $G_{s}(\mathbf{r}, t)$ [21], which differs at $|t| \rightarrow 0$ from the solution of the diffusion equation. Respectively, it provides, in principle, deviation of the function $\Delta \varepsilon\left(Q^{2}\right)$ from a straight line. But this is the case only for large diffusing molecules or liquids with high viscosity.

Random jumps of atoms in a liquid occur, like those in a crystal, from time to time between neighbouring sites. In simple liquids this results from their quasi-crystalline structure [22-24]. The function $\Delta \varepsilon\left(Q^{2}\right)$, provided only by jump diffusion, represents nonlinear dependence $[25,26]$, but it is saturated at high $Q^{2}$, when $\Delta \varepsilon$ becomes constant $\sim 1 / \tau_{0}$, where $\tau_{0}$ is the mean residence time of the atom in a site between successive jumps. But this contradicts observations. Therefore, both continuous and jump diffusion have to be taken into account simultaneously.

A number of attempts have been made to unify these limiting approaches to the dynamics. In particular, Singvi and Sjölander [25] supposed that the atom vibrated for some time in any site, then it crawled to another one. This process is specified by the mean residence time $\tau_{0}$ and the mean time of the continuous motion between sites $\tau_{1}$. For $\tau_{1} \gg \tau_{0}$ the authors have been getting pure continuous diffusion, and for $\tau_{1} \ll \tau_{0}$ pure jumps. However, it seems strange that vibrations alternate with continuous motion.

A more satisfactory approach was advanced by Oscotskii [27], who made the following assumption. The sites, between which the atoms are jumping, take part in a continuous diffusion, which proceeds simultaneously with jumps. The equations for the QENS peak broadening, derived in [27], beautifully explained the experimental data of $[17-20]$.

From our point of view, the assumption of Oscotskii is tightly bound to Fisher's idea [28] that interacting atoms (molecules) in a liquid form aggregates (Lagrange particles), continuously diffusing throughout the liquid (see also [29]). In particular, such a particle can be a single molecule. Then the Lagrange particle may be treated as a carrier of light atoms, which make random jumps inside it between adjacent sites. Developing these ideas, we shall consider in this paper simultaneously the influence of both types of continuous motion of Lagrange particle (translational and rotational) along with jumps of atoms on the broadening of QENS peak.

\section{THEORY}

As usuall [2-20], we shall take into consideration only the incoherent scattering of neutrons by hydrogen atoms in a liquid, since the incoherent scattering cross section of neutrons by protons $\sigma_{\mathrm{H}}$ is much larger than that for other isotopes. Then the double differential incoherent scattering cross section of neutrons by a liquid is determined by $[1,30]$

$$
\left(\frac{d^{2} \sigma}{d \Omega d E^{\prime}}\right)_{\mathrm{inc}}=A \frac{1}{2 \hbar} \int_{-\infty}^{\infty} d t e^{-i \omega t} F_{s}(\mathbf{Q}, t)
$$

where 


$$
A=N \frac{k^{\prime}}{k} \frac{\sigma_{H}}{4 \pi^{2}}
$$

$N$ is the number of hydrogen atoms in the liquid, $\mathbf{k}$ and $\mathbf{k}^{\prime}$ are the wave vectors of incident and scattered neutrons respectively, $E$ and $E^{\prime}$ are the corresponding kinetic energies of the neutrons, $\mathbf{Q}=\mathbf{k}-\mathbf{k}^{\prime}$ represents the wave vector transfer and $\hbar \omega=E-E^{\prime}$ is the energy transfer.

The intermediate scattering function

$$
F_{s}(\mathbf{Q}, t)=\left\langle e^{-i \mathbf{Q R}(0)} e^{i \mathbf{Q R}(t)}\right\rangle
$$

is the Fourier transform of the Van Hove function $G_{s}(\mathbf{r}, t)$ for one of the hydrogen atoms, whose position is determined by the radius-vector $\mathbf{R}$. Its time-dependent value in (3) stands for the operator in the Heisenberg representation. The brackets $\langle\ldots\rangle$ denote the statistical as well as quantum-mechanical averaging over the initial states of the scatterer.

Let the energy distribution of neutrons emitted by a source be approximated by the Lorentzian

$$
w_{s}(E)=\frac{\Gamma_{s} / 2 \pi}{\left(E-E_{0}\right)^{2}+\left(\Gamma_{s} / 2\right)^{2}}
$$

with $E_{0}=\hbar^{2} k_{0}^{2} / 2 m$, where $m$ is the neutron mass. Besides, the energy resolution function of the neutron detector is assumed to be

$$
w_{d}\left(E^{\prime}\right)=\frac{\Gamma_{d} / 2 \pi}{\left(E^{\prime}-E_{0}^{\prime}\right)^{2}+\left(\Gamma_{d} / 2\right)^{2}} .
$$

The experimentally measured cross section is determined by the convolution

$$
\sigma_{\mathrm{sc}}(\varepsilon)=\int_{0}^{\infty} d E w_{s}(E) \int_{0}^{\infty} d E^{\prime} w_{d}\left(E^{\prime}\right)\left(\frac{d^{2} \sigma}{d \Omega d E^{\prime}}\right)_{\mathrm{inc}} .
$$

Eor $\Gamma_{s} \ll E_{0}$ and $\Gamma_{d} \ll E_{0}^{\prime}$ it will be given by

$$
\sigma_{\mathrm{SC}}(\varepsilon)=A \int_{0}^{\infty} \frac{d t}{\hbar} \cos (\varepsilon t / \hbar) e^{-\Gamma_{\mathrm{o}} t / 2 \hbar} F_{s}(\mathbf{Q}, t)
$$

where

$$
\Gamma_{0}=\Gamma_{s}+\Gamma_{d}, \quad \varepsilon=E_{0}-E_{0}^{\prime}, \quad \mathbf{Q}=\mathbf{k}_{0}-\mathbf{k}_{0}^{\prime},
$$

and $\mathbf{k}_{0}, \mathbf{k}_{0}^{\prime}$ are associated with mean energies $E_{0}, E_{0}^{\prime}$. The integrand in (7) has significant value in the time interval $0 \leq t \sim t_{0}$, where $t_{0}=\hbar / \Gamma_{0}$ may be treated as a collision time of the neutron with a target in the absence of diffusion. It plays the same role as the nuclear lifetime in the theory of Mössbauer effect in Brownian particles [31-35].

Let us write the radius-vector of the hydrogen atom as

$$
\mathbf{R}=\mathbf{R}^{(0)}+\mathbf{u}
$$

where $\mathbf{R}^{(0)}$ determines the equilibrium position of the atom in the laboratory coordinate frame and $\mathbf{u}$ is its displacement from this position due to vibrations. By assumption, each atom is a part of any Lagrange particle, whose center is determined by $\mathbf{R}_{L}$. Then it is useful to write

$$
\mathbf{R}^{(0)}=\mathbf{R}_{L}+\mathbf{r}
$$

where $\mathbf{r}$ indicates the position of the proton in the center of mass system $x, y, z$ with origin in the center of Lagrange particle and axis $z$ directed along the vector Q.

The intermediate function for the spherical Lagrange particle of the radius $R$ takes the form

$$
F_{s}(\mathbf{Q}, t)=F_{s}^{(t)}(\mathbf{Q}, t) F_{s}^{(r, j)}(\mathbf{Q}, t) F_{s}^{(v)}(\mathbf{Q}, t)
$$

where the factor $F_{s}^{(t)}$ describes the translational motion, $F_{s}^{(r, j)}$ is due to rotation and jumps, $F_{s}^{(v)}$ to vibrations of the hydrogen in one of the sites. They are given by Eq. (3) with $\mathbf{R}$ replaced by $\mathbf{R}_{L}$ for $F^{(t)}$ or by $\mathbf{r}$ for $F^{(r, j)}$ and by $\mathbf{u}$ for $F^{(v)}$.

The quantum vibrations are described by a standard expression (see, e.g., [30]):

$$
F_{s}^{(v)}(\mathbf{Q}, t)=e^{-2 W(\mathbf{Q})} \exp (\langle\mathbf{Q} \cdot \mathbf{u}(0) \mathbf{Q} \cdot \mathbf{u}(t)\rangle)
$$

where $\exp (-2 W(\mathbf{Q}))$ is the Debye-Waller factor. The correlator in (12) defines all possible processes with creation or annihilation of phonons. For quasi-elastic scattering, being the phononless process, $F_{s}^{(v)}=e^{-2 W(\mathbf{Q})}$. All other types of the hydrogen movement we shall treat in classical approximation.

The Langevin equation for the translational Brownian motion gives at $t>0$ the well known intermediate scattering function [31]

$$
F_{s}^{(t)}(\mathbf{Q}, t)=\exp \left\{-a\left(\beta t-1+e^{-\beta t}\right)\right\}
$$

where

$$
a=\frac{Q^{2} D_{0}}{\beta}, \quad D_{0}=\frac{k_{B} T}{6 \pi R \eta}, \quad \beta=\frac{6 \pi R \eta}{M},
$$

$D_{0}$ is the translational diffusion coefficient of the sphere in a liquid of viscosity $\eta$ and temperature $T, k_{B}$ stands for the Boltzmann constant, $M$ is the mass of Lagrange particle. When $t \gg \beta^{-1}$ (13) simplifies to the function

$$
F_{s}^{(t)}(\mathbf{Q}, t)=\exp \left(-Q^{2} D t\right)
$$

resulting also from the diffusion equation. Therefore, $\beta^{-1}$ 
means a characteristic time for the continuous translational diffusion in the liquid.

In classical approach the function $F_{s}^{(r, j)}$ takes the form

$$
F_{s}^{(r, j)}(\mathbf{Q}, t)=\left\langle e^{i \mathbf{Q}(\mathbf{r}(t)-\mathbf{r}(0))}\right\rangle
$$

where the brackets denote only the statistical averaging. For separation of the Brownian rotation and jumps we introduce one more coordinate frame $\xi, \eta, \zeta$, rotating together with the Lagrange particle. Its origin is placed in the particle's center and axes are rigidly bound to it. The unit vectors along the axes $x, y, z$ and $\xi, \eta, \zeta$ are denoted by $\mathbf{e}_{1}, \mathbf{e}_{2}, \mathbf{e}_{3}$ and $\mathbf{e}^{\prime}{ }_{1}(t), \mathbf{e}^{\prime}{ }_{2}(t), \mathbf{e}^{\prime}{ }_{3}(t)$ respectively. Let in the initial moment $t=0$ they coincide, $\mathbf{e}_{i}^{\prime}(0)=\mathbf{e}_{i}$.

The position of the atom in the Lagrange particle $\mathbf{r}$ may change from time to time due to random jumps in the neighbouring sites. When jumps are absent, at any moment its coordinates $\xi_{\alpha}(t)=\{\xi, \eta, \zeta\}$ with respect to the axes $\xi, \eta, \zeta$ do not change, i.e., $\xi_{\alpha}(t)=\xi_{\alpha}(0)$. Jumps lead to the displacement specified by coordinates $\Delta \xi_{\alpha}(t)=\xi_{\alpha}(t)-\xi_{\alpha}(0)$. The vector $\mathbf{r}$ may be now represented as

$$
\begin{aligned}
\mathbf{r}(t) & =r_{0}\left\{\sin \theta_{0}\left(\cos \phi_{0} \mathbf{e}_{1}{ }_{1}(t)+\sin \phi_{0} \mathbf{e}_{2}^{\prime}(t)\right)\right. \\
& \left.+\cos \theta_{0} \mathbf{e}_{3}^{\prime}(t)\right\}+\sum_{\alpha=1}^{3} \Delta \xi_{\alpha}(t) \mathbf{e}_{\alpha}^{\prime}(t),
\end{aligned}
$$

where $r_{0}, \theta_{0}, \phi_{0}$ are the spherical coordinates of the vector $\mathbf{r}$ at $t=0$. In general case the rotation and jumps are mixed processes, but situation simplifies in the approximation of small rotations [34,35]. Really, the meansquare angle $\left\langle\vartheta^{2}\right\rangle^{1 / 2}$ of the Brownian rotation during time $t$ is defined by

$$
\left\langle\vartheta^{2}\right\rangle=2 D_{r} t
$$

where $D_{r}=k_{B} T / 8 \pi R^{3} \eta$ is the rotational diffusion coefficient. In our experiment with $t_{0} \sim 1 \mathrm{ps}, R \sim 3 \AA$, $\eta \sim 0.01 \mathrm{~g} / \mathrm{cm} \cdot \mathrm{s}$ one has $\left\langle\vartheta^{2}\right\rangle \sim 0.01$.

Any small rotation is a product of three rotations to angles $\vartheta_{1}, \vartheta_{2}, \vartheta_{3}$ about the axes $x, y, z$ respectively. As a consequence, the unit vectors $\mathbf{e}_{i}$ are related to $\mathbf{e}_{i}^{\prime}$ by

$$
\begin{aligned}
& \mathbf{e}_{1}^{\prime} \approx \mathbf{e}_{1}+\vartheta_{3} \mathbf{e}_{2}-\vartheta_{2} \mathbf{e}_{3}, \\
& \mathbf{e}_{2}^{\prime} \approx-\vartheta_{3} \mathbf{e}_{1}+\mathbf{e}_{2}+\vartheta_{1} \mathbf{e}_{3}, \\
& \mathbf{e}_{3}^{\prime} \approx \vartheta_{2} \mathbf{e}_{1}-\vartheta_{1} \mathbf{e}_{2}+\mathbf{e}_{3} .
\end{aligned}
$$

The condition $\left\langle\vartheta^{2}\right\rangle^{1 / 2} \ll 1$ allows us also to put the angles $\vartheta_{\alpha}$ to vary from $-\infty$ to $+\infty$. Estimations show that $\Delta \xi_{\alpha}\left(t_{0}\right) / r_{0} \sim\left\langle\Delta \xi_{\alpha}^{2}\right\rangle^{1 / 2} / R \ll 1$, i. e., the displacement of the atom, associated with jumps during the collision time $t_{0}$, is much less than $R$. Then, substituting (19) into (17) and neglecting terms of the second order in parameters $\vartheta$ and $\Delta \xi / R$, one gets

$$
\begin{aligned}
\mathbf{Q} \cdot(\mathbf{r}(t)-\mathbf{r}(0)) & =Q r_{0} \sin \theta_{0}\left(-\cos \phi_{0} \vartheta_{2}+\sin \phi_{0} \vartheta_{1}\right) \\
& +Q \Delta \zeta(t)
\end{aligned}
$$

Inserting (20) into (16) one finds $F_{s}^{(r, j)}(\mathbf{Q})$ as a product of $F_{s}^{(r)}(\mathbf{Q})$ and $F_{s}^{(j)}(\mathbf{Q})$.

In particular, the rotational function becomes

$$
\begin{aligned}
F_{s}^{(r)}(\mathbf{Q}, t) & =\int_{-\infty}^{\infty} d \vartheta_{1} \int_{-\infty}^{\infty} d \vartheta_{2} \int_{-\infty}^{\infty} d \vartheta_{3} \\
& \times \exp \left[i Q r_{0} \sin \theta_{0}\left(-\cos \varphi_{0} \vartheta_{2}+\sin \varphi_{0} \vartheta_{1}\right)\right] \\
& \times W\left(\left\{\vartheta_{\alpha}\right\} ; t\right)
\end{aligned}
$$

Here $W\left(\left\{\vartheta_{\alpha}\right\} ; t\right)$ is the probability density that the orientation of the axes $\xi, \eta, \zeta$ at the moment $t$ is determined by the angles $\left\{\vartheta_{\alpha}\right\}$, if at $t=0$ all the angles $\vartheta_{\alpha}=0$.

It satisfies the initial condition

$$
W\left(\vartheta_{\alpha} ; 0\right)=\delta\left(\vartheta_{1}\right) \delta\left(\vartheta_{2}\right) \delta\left(\vartheta_{3}\right)
$$

A stochastic Langevin-type equation for the rotational diffusion may be written as

$$
\frac{d \omega}{d t}=-\beta_{r} \omega+\mathbf{M}(t) / \mathcal{J}
$$

where $\omega_{\alpha}=\dot{\vartheta}_{\alpha}$ are the components of the angular velocity $\omega$ along the axes $x, y, z ; \mathbf{M}(t)$ are momenta of random forces acting on the particle, $\beta_{r}=8 \pi R^{3} \eta / \mathcal{J}$; $\mathcal{J}=0.4 M R^{2}$ is the moment of inertia of the sphere with mass $M$ and radius $R$. Eq. (23) is similar to Langevin equation for the translational motion whose solution is well known [36]. Now $\mathcal{J}$ plays the role of the mass $M$ and $\left\{\vartheta_{\alpha}\right\}$ are analogous to linear coordinates $\mathbf{r}$. Then the solution of Eq. (23) may be written in full analogy with the well known solution of Langevin equation for the translational motion [36]:

$$
\begin{aligned}
W\left(\left\{\vartheta_{\alpha}\right\} ; t\right) & =\left(4 \pi q \int_{0}^{t} \psi_{r}^{2}(x) d x\right)^{-3 / 2} \\
& \times \exp \left\{-\frac{\sum_{\alpha=1}^{3}\left(\vartheta_{\alpha}-\omega_{\alpha}(0) \psi_{r}(0)\right)^{2}}{4 q \int_{0}^{t} \psi_{r}^{2}(x) d x}\right\},
\end{aligned}
$$

where $\omega(0)$ is the angular velocity at $t=0$, and

$$
\begin{array}{r}
q=\beta_{r} k_{B} T / \mathcal{J}, \quad \psi_{r}(0)=\frac{1}{\beta_{r}}\left(1-e^{-\beta_{r} t}\right) \\
\int_{0}^{t} \psi_{r}^{2}(x) d x=\frac{1}{2 \beta_{r}^{3}}\left(2 \beta_{r} t-3+4 e^{-\beta_{r} t}-e^{-2 \beta_{r} t}\right) .
\end{array}
$$


After substitution of (24) into (21) and averaging over initial angular velosity distribution

$$
P(\omega(0))=\left(\frac{\mathcal{J}}{2 \pi k_{B} T}\right)^{3 / 2} \exp \left\{-\frac{\mathcal{J} \omega^{2}(0)}{2 k_{B} T}\right\}
$$

one gets immediately

$$
F_{s}^{(r)}(\mathbf{Q}, t)=\exp \left\{-a_{r}\left(\beta_{r} t-1+e^{-\beta_{r} t}\right)\right\}
$$

where

$$
a_{r}=Q^{2} D_{r} r_{0}^{2} \sin ^{2} \theta_{0} / \beta_{r}
$$

By definition, $\beta_{r}^{-1}=0.3 \beta^{-1}$ and

$$
a_{r}=0.225 a\left(r_{0} / R\right)^{2} \sin ^{2} \theta_{0} \ll a .
$$

The intermediate function for the jump diffusion in the limit $\tau_{1} \rightarrow 0$ is given by

$$
F_{s}^{(j)}(\mathbf{Q}, t)=e^{-\Delta \Gamma_{j} t / 2 \hbar}
$$

with the broadening [25]

$$
\Delta \Gamma_{j}=\frac{2 \hbar}{\tau_{0}}\left[1-e^{-2 W(\mathbf{Q})}\left(1+Q^{2} D_{1} \tau_{0}\right)^{-1}\right]
$$

where $\tau_{0}$ is the mean residence time of the proton in a site between successive jumps and the jump diffusion coefficient $D_{1}$ is related to the mean-square jump length $l_{0}$ by

$$
D_{1}=l_{0}^{2} / 6 \tau_{0}
$$

Multiplying (12), (13), (27) and (30) one has the following expression for the QENS cross section:

$$
\sigma_{\mathrm{sc}}(\varepsilon)=A e^{a} e^{-2 W} \operatorname{Re} \int_{0}^{\infty} \frac{d t}{\hbar} e^{i \varepsilon t / \hbar-\left(\Gamma_{0}+\Delta \Gamma_{j}\right) t / 2 \hbar-a \beta t-a_{r} \beta_{r} t} \exp \left\{-a e^{-\beta t}\right\} \exp \left\{a_{r}\left(1-e^{-\beta_{r} t}\right)\right\} .
$$

The cross section should be yet averaged over the initial positions of the hydrogen atoms inside the Lagrange particle, i. e., over coordinates $r_{0}, \theta_{0}$. Using the condition (29), we shall replace in (33) the exponent $\exp \left\{a_{r}\left(1-e^{-\beta_{r} t}\right)\right\}$ by unity. Expanding another exponent $\exp \left\{-a e^{-\beta t}\right\}$ in power series and averaging (33) over uniform distribution of hydrogen atoms one gets

$$
\bar{\sigma}_{\mathrm{sc}}(\varepsilon)=\frac{4 A e^{a} e^{-2 W}}{\hbar Q^{2} D_{0}} \sum_{n=0}^{\infty}(-1)^{n} \frac{a^{n}}{n !} \operatorname{Re}\left\{\sqrt{1+1 / x_{n}(\varepsilon)} \operatorname{Arsh} \sqrt{x_{n}(\varepsilon)}-1\right\}
$$

where

$$
x_{n}(\varepsilon)=\frac{3}{4} \frac{\hbar Q^{2} D_{0}}{\left(\Gamma_{0}+\Delta \Gamma_{j}\right) / 2+\hbar Q^{2} D_{0}+n \hbar \beta+i \varepsilon} .
$$

The effective width of this QENS peak may be defined as $[37]$

$$
\Gamma_{\text {eff }}=\frac{2}{\pi \bar{\sigma}_{\mathrm{sc}}(0)} \int_{-\infty}^{\infty} d \varepsilon \bar{\sigma}_{\mathrm{sc}}(\varepsilon)
$$

Substitution of (34) into (36) gives (see also [34])

$$
\Gamma_{\mathrm{eff}}=\hbar Q^{2} D_{0} e^{-a}\left\{2 \sum_{n=0}^{\infty} \frac{a^{n}}{n !}\right.
$$

$$
\left.\times(-1)^{n}\left[\sqrt{1+1 / x_{n}(0)} \operatorname{Arsh} \sqrt{x_{n}(0)}-1\right]\right\}^{-1}
$$

Langevin's corrections to the diffusion theory become essential if $a \sim 1$. This condition is equivalent to $\Gamma \sim \hbar \beta$. In the opposite case, when $a \ll 1$, expansion (34) reduces to single term with $n=0$. If $\hbar Q^{2} D_{0} \ll \Gamma_{0}$ it simplifies to the Lorentzian function

$$
\bar{\sigma}_{s c}(\varepsilon)=A e^{-2 W} \frac{(\Gamma / 2)^{2}}{\varepsilon^{2}+(\Gamma / 2)^{2}},
$$

where the width is

$$
\begin{aligned}
& \Gamma=\Gamma_{0}+\Delta \Gamma, \quad \Delta \Gamma=\Delta \Gamma_{\text {coll }}+\Delta \Gamma_{j}, \\
& \Delta \Gamma_{\text {coll }}=2.6 \hbar Q^{2} D_{0} .
\end{aligned}
$$




\section{EXPERIMENT}

The QENS measurements were carried out at the reactor of the Institute for Nuclear Research in Kyiv using multidetector time-of-flight spectrometer. A schematic layout of this spectrometer is displayed in Fig. 1. The flux density of cold neutrons reflected from the monochromating crystal ( $\mathrm{Pb}, \mathrm{Cu}, \mathrm{Zn}$ ) amounts $2 \cdot 10^{8} \mathrm{n} \mathrm{cm}^{-2} \mathrm{~s}^{-1}$ for the average neutron energy $E=0.25 \mathrm{meV}$. This value of the flux varies depending on the type of the monochromating crystal and neutron energy. In our experiment we have been using neutrons with $E=13.1 \mathrm{meV}$ (the wavelength $\lambda=2.50 \AA$ ). The monochromated neutrons pass through the chopper, being a rotor spinning with high angular velocity, to produce a pulsed neutron beam. The flux density of this pulsed beam is $4 \cdot 10^{3} \mathrm{n} \mathrm{cm}^{-2} \mathrm{~s}^{-1}$. The energies of scattered neutrons are determined by measuring the time of flight of scattered neutrons over $2.87 \mathrm{~m}$ flight path between the sample and detectors. Eight detectors filled with ${ }^{3} \mathrm{He}$ under the pressure $10 \mathrm{~atm}$ were installed at the scattering angles $\theta_{1}=25.1^{\circ}, \theta_{2}=40.5^{\circ}, \theta_{3}=55.7^{\circ}, \theta_{4}=$ $70.9^{\circ}, \theta_{5}=86.1^{\circ}, \theta_{6}=101.3^{\circ}, \theta_{7}=116.5^{\circ}, \theta_{8}=-9.5^{\circ}$ with respect to the incident neutron beam. We made measurements using first five detectors. Even in this case the wave vector transfer $Q=(4 \pi / \lambda) \sin (\theta / 2)$ varies from $1.09 \AA^{-1}$ to $3.43 \AA^{-1}$ as $\lambda=2.5 \AA$.

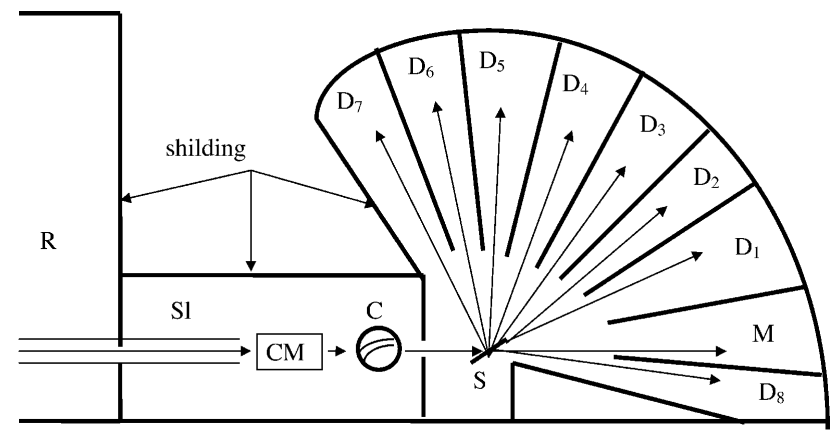

Fig. 1. Scheme of the time-of-flight spectrometer. The abbreviation $\mathrm{R}$ designates the reactor, $\mathrm{CM}$ - the crystal monochromating neutrons, $\mathrm{C}$ - the chopper, $\mathrm{S}$ - the sample, $\mathrm{M}$ - the monitor, $D_{i}$ - the detectors installed at the angles $\theta_{1}=25.1^{\circ}, \theta_{2}=40.5^{\circ}, \theta_{3}=55.7^{\circ}, \theta_{4}=70.9^{\circ}$, $\theta_{5}=86.1^{\circ}, \theta_{6}=101.3^{\circ}, \theta_{7}=116.5^{\circ}, \theta_{8}=-9.5^{\circ}$ with respect to the transmitting neutron beam.

The energies of neutrons scattered to different directions are measured simultaneously by means of the electronic system of multi-dimensional analysis. The energy resolution function was found by a standard vanadium calibration run. Its full width at half maximum $\Gamma_{0}$, depending on $Q$, ranges from $0.78 \mathrm{meV}$ at small $Q$ to $1.00 \mathrm{meV}$ at large $Q$.

The QENS spectra (see Figs. 2-4)were taken at room temperature $T=293 \mathrm{~K}$ for three types of spirit (ethanol: $\mathrm{C}_{2} \mathrm{H}_{5} \mathrm{OH}$; propanol: $\mathrm{C}_{3} \mathrm{H}_{7} \mathrm{OH}$; n-nonanol: $\mathrm{C}_{9} \mathrm{H}_{19} \mathrm{OH}$ ). General tendency of the observed spectra is that the QENS peak broadens and its intensity falls with increas- ing of the scattering angle. At large angles the peak is hardly separated from the background, therefore, the limiting angle is taken $\theta_{\max }=86.1^{\circ}$.

\section{EXPERIMENTAL RESULTS}

Fitting the intensities of the QENS peaks, which are proportional to the Debye-Waller factor $e^{-2 W}$, we found the mean-square vibrational amplitudes of hydrogen atoms $\left\langle u_{x}^{2}\right\rangle^{1 / 2}$. They are practically the same for all spirits, $\left\langle u_{x}^{2}\right\rangle \simeq 0.2 \AA^{2}$. More exact values are displayed in Table 1 .

\begin{tabular}{|l|l|l|l|}
\hline sample & $\left\langle u_{x}^{2}\right\rangle, \AA^{2}$ & $l_{0}, \AA$ & $\tau_{0}, \mathrm{ps}$ \\
\hline ethanol & 0.184 & 0.8 & 1.4 \\
\hline propanol & 0.176 & 1.02 & 2.43 \\
\hline n-nonanol & 0.172 & 1.05 & 2.38 \\
\hline
\end{tabular}

Table 1. The fitting parameters.

\begin{tabular}{|l|l|l|l|l|l|}
\hline sample & $D_{0}$ & $D_{1}$ & $D$ & $\eta$ & $R$ \\
\hline ethanol & 0.63 & 0.76 & 1.39 & 1.20 & 2.85 \\
\hline propanol & 0.31 & 0.71 & 1.02 & 2.23 & 3.10 \\
\hline n-nonanol & 0.08 & 0.77 & 0.85 & 6.32 & 4.10 \\
\hline
\end{tabular}

Table 2. The diffusion coefficients (in units of $10^{-5} \mathrm{~cm}^{2} \mathrm{~s}^{-1}$ ) together with the viscosities of spirits (in units of $10^{-2} \mathrm{~g} \mathrm{~cm}^{-1} \mathrm{~s}^{-1}$ ) and radii of molecules (in $\AA$ ).

From data shown in Figs. 2-4 we determined the width at half maximum of the QENS peaks for five points of $Q^{2}$. Extracting then the width of the energy resolution function, the broadenings of the lines were found at half maximum $\Delta \varepsilon$, which are represented in Fig. 5 .

We assume the spirit molecules to diffuse undependently of each other, i.e., the Lagrange particle consists only of single molecule. The molecular radius $R$ is then estimated with the aid of trivial relation $M=$ $\left(4 \pi R^{3} / 3\right) \rho$, where $M$ is the mass of the molecule and $\rho$ is the density of the liquid. These values of radii together with viscosities completely define the translational diffusion coefficients $D_{0}$. The functions $\Delta \varepsilon$ manifest typical nonlinear dependence on $Q^{2}$. Since in our experiment $a \ll 1$, the curvature of the observed function $\Delta \varepsilon\left(Q^{2}\right)$ may be explained by the common influence of jumps and continuous diffusion. At small $Q$ both mechanisms of diffusion give contributions to $\Delta \Gamma$ linearly depending on $Q^{2}$, according to Eqs. (31), (36). Although at high $Q$ the jump diffusion is "frosen", giving $\Delta \Gamma_{j}=2 \hbar / \tau_{0}$ as $e^{-2 W(Q)} \simeq 0$, the contribution of continuous motion grows proportionally to $Q^{2}$. 


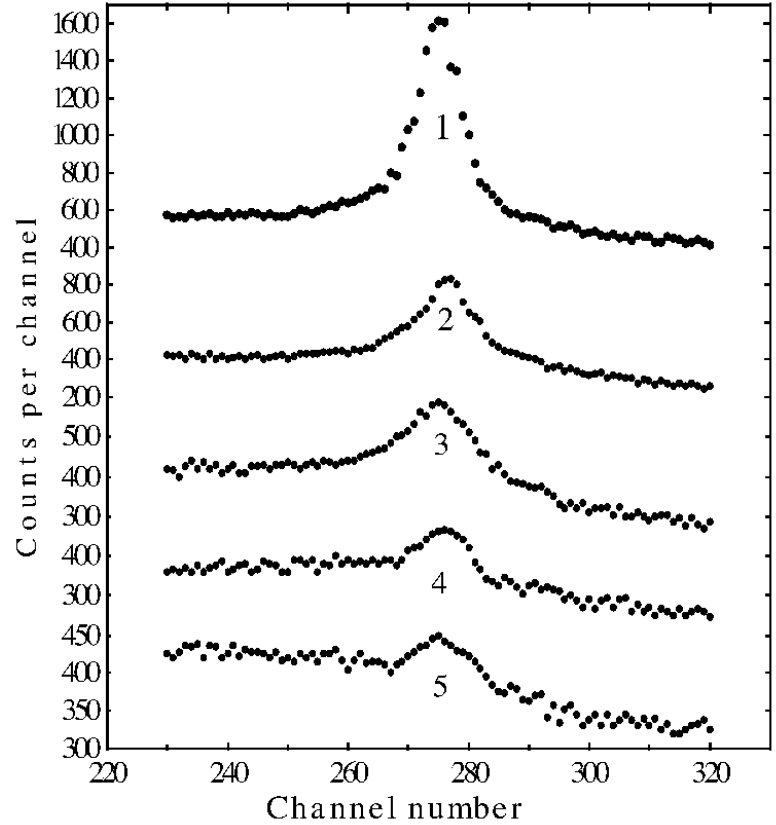

Fig. 2. The QENS spectra of the ethanol. The numbers at the curves $i$ indicate the scattering angles $\theta_{i}$.

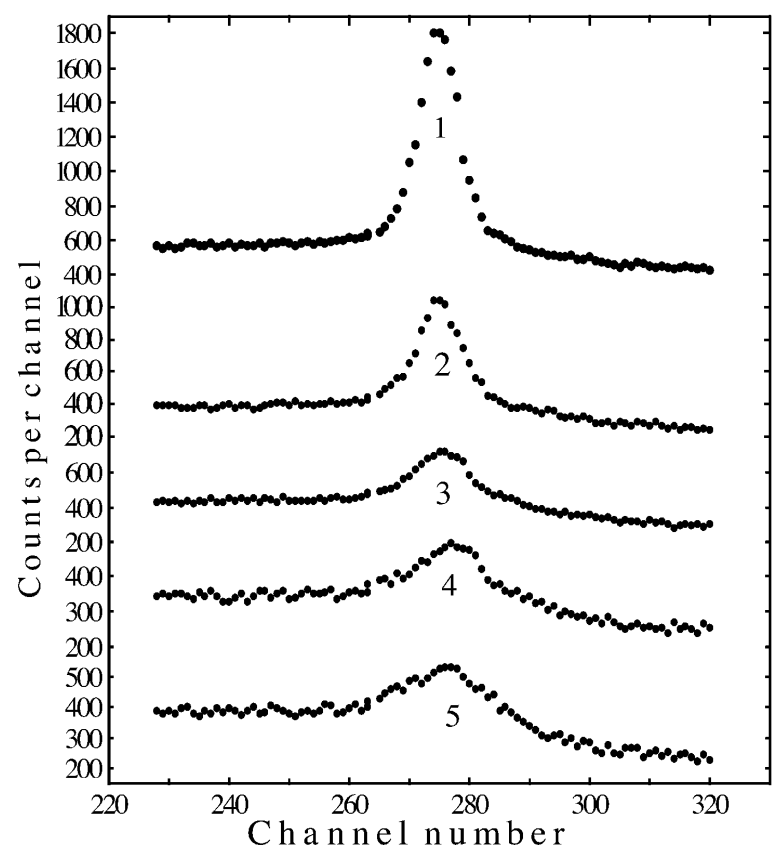

Fig. 3. The QENS spectra of the propanol.

Our data were fitted with the aid of two parameters (the residence time of the atom $\tau_{0}$ and the average jump length $l_{0}$ ). They are listed in Table 1 . Using these parameters we calculated the jump diffusion coefficients $D_{1}=l_{0}^{2} / 6 \tau_{0}$, which are given in Table 2 together with the translational diffusion coefficients $D_{0}$, total diffusion coefficients $D=D_{0}+D_{1}$, radii and viscosities of liquids.

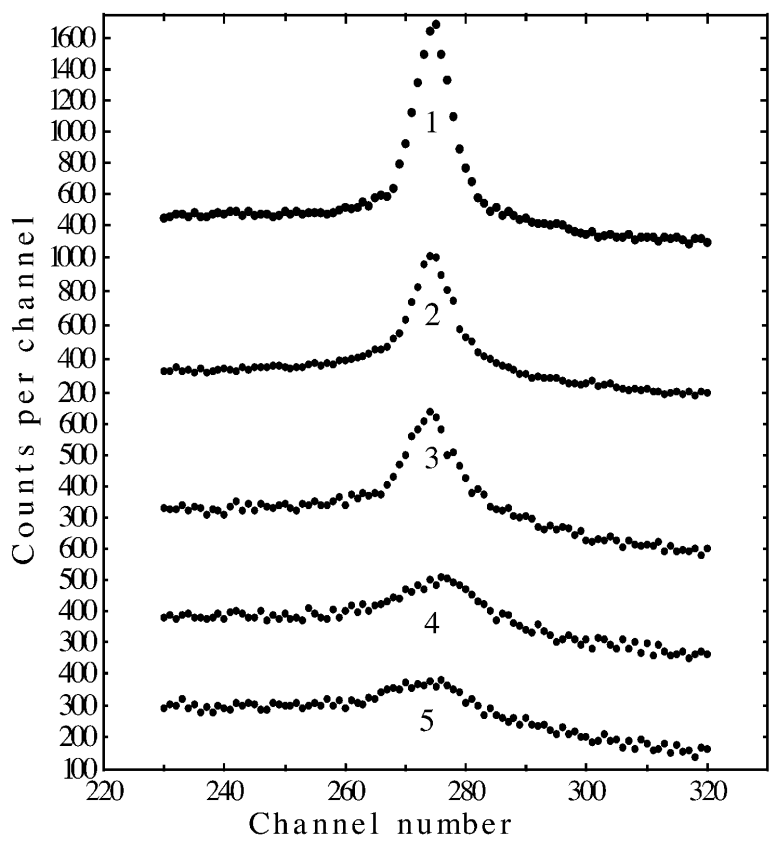

Fig. 4. QENS spectra of the normal nonanol.

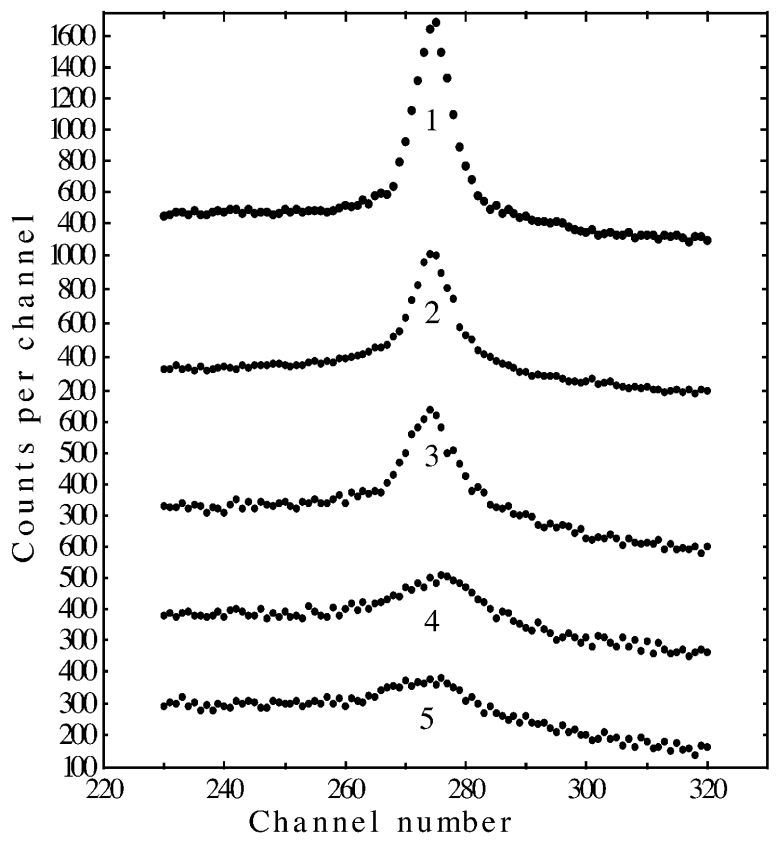

Fig. 5. The broadening of the QENS peak versus the square of the wave vector transfer. The dots are the experimental data, the solid line is the fitting curve by Eq. (38).

\section{DISCUSSION}

Thus, we analyzed the combined influence of different diffusion mechanisms on the QENS spectra. Previously 
their role has been considered separately. So, only Brownian rotation without jumps was treated in [38], while the translational diffusion + jumps were regarded in [27] but without rotation. It should be noted that these theories were based on the diffusion equations, whereas we start from the most correct Langevin equations. We seem to be the first to develop such an approach for the rotational motion. It is done in the approximation of small rotations, in which the situation resembles the translational case. Namely, the corresponding solutions (13) and (27) are very similar. We derived general equation (33) for the QENS cross section, which can be calculated numerically. It is found that the Langevin corrections for rotation are less essential than those for the translational motion since $a_{r} \ll a$. Thanks to this enequality the rotation can be treated on the basis of the diffusion equa- tion only, while the Langevin equation is conserved for the translational motion. It allowed us to get the analitycal expression (34) for the QENS cross section as an expansion in powers of the parameter $a$. When $a \ll 1$ and the broadening of the line $\Delta \Gamma$ is small it reduces to the Lorentzian function (38), which resembles the result of Oscotskii [27]. However, our equation contains additional contribution to the broadening of the QENS peak $0.6 \hbar Q^{2} D_{0}$ due to rotation. Only in the same limiting case the separation of single-particle (jump) motion from the collective one becomes an easy task since $\Delta \Gamma_{j}$ and $\Delta \Gamma_{\text {coll }}$ are characterized by different dependence on $Q^{2}$ (see also [29]). All these calculations are well confirmed by our observations.

Authors are grateful to professors L. A. Bulavin and A. V. Zatovsky for helpful discussions.
[1] I. I. Gurevich, L. V. Tarasov, Fizika neitronov nizkikh energij (Physics of Low Energy Neutrons) (Nauka, Moscow, 1965).

[2] J. Teixeira, M.-C. Bellissent-Funel, Phys. Rev. A 31, 1913 (1985).

[3] J. Teixeira, M.-C. Bellissent-Funel, S. H. Chen, B. Dorner, Phys. Rev. Lett. 54, 2681 (1985).

[4] P. S. Salmon, M.-C. Bellissent-Funel, G. J. Herdman, J. Phys.: Cond. Matt. 2, 4297 (1990).

[5] J. Teixeira, M.-C. Bellissent-Fumel, S. H. Chen, J. Phys. Cond. Matt. 2, SA105 (1990).

[6] J. Teixeira, M.-C. Bellissent-Funel, S. H. Chen, J. Mol. Liq. 48, 123 (1991).

[7] L. A. Bulavin, A. A. Vasilkevich, A. K. Dorosh, P. G. Ivanitsky, V. T. Krotenko, V. I. Slisenko, Ukr. Phys. J. 31, 1703 (1986).

[8] V. I. Slisenko, P. G. Ivanitsky, T. V. Karmazina, N. A. Klimenko, V. T. Krotenko, A. A. Vasilkevich, A. N. Maistrenko, Ukr. Chem. J. 59, 259 (1993).

[9] W. Kunz, P. Turg, M.-C. Bellissent-Funel, P. Calmettes, J. Chem. Phys. 95, 6902 (1991).

[10] F. Aliotta, M.-C. Bellissent-Funel, D. I. Donato, P. Migliardo, C. Vasi, Physica B, 180-181, 861 (1992).

[11] W. Kunz, P. Calmettes, M.-C. Bellissent-Funel, J. Chem. Phys. 99, 2079 (1993).

[12] S. Magazu, Physica B 226, 92 (1996).

[13] M.-C. Bellissent-Funel, K. F. Bradley, S. H. Chen, J. Lal, J. Teixeira, Physica A 201, 277 (1993).

[14] M.-C. Bellissent-Funel, S. H. Chen, J.-M. Zanotti, Phys. Rev. E 51, 4558 (1995).

[15] M.-C. Bellissent-Funel, J. Mol. Liq. 78, 19 (1998).

[16] J.-M. Zanotti, M.-C. Bellissent-Funel, J. Parello, Biophys. J. 76, 2390 (1999).

[17] A. G. Novikov, M. N. Rodnikova, V. V. Savostin,
O. V. Sobolev, Chem. Phys. Lett. 259, 391 (1996).

[18] A. G. Novikov, M. N. Rodnikova, V. V. Savostin, O. V. Sobolev, Physica B 234-236, 340 (1997).

[19] A. Novikov, M. Rodnikova, J. Barthel, O. Sobolev, J. Mol. Liq. 79, 203 (1999).

[20] A. Novikov, M. Rodnikova, V. Savostin, O. Sobolev, J. Mol. Liq. 82, N1-2 (1999).

[21] L. Van Hove, Phys. Rev. 95, 249 (1954).

[22] J. Frenkel, Kinetic Theory of Liquids (Oxford University Press, New York, 1946).

[23] J. L. Jones, J. A. Pople, Proc. Roy. Soc. (London) A 205, 155 (1951).

[24] H. S. Frank, Proc. Roy. Soc. (London) A 247, 481 (1958).

[25] K. S. Singwi, A. Sjölander, Phys. Rev. 119, 863 (1960).

[26] C. T. Chudley, R. J. Elliot, Proc. Phys. Soc. (London) 77, 353 (1961).

[27] V. S. Oskotskii, Fiz. Tverd. Tela 5, 1082 (1963).

[28] I. Z. Fisher, Zh. Eksp. Teor. Fiz. 61, 1617 (1971).

[29] V. Ya. Antonchenko, A. S. Davydov, V. V. Il'yn, Fizika vody (Water physics) (Naukova Dumka, Kiev, 1991)

[30] Dynamics of Solids and Liquids by Neutron Scattering, edited by S. W. Lovesey, T. Springer (Springer Verlag, Berlin Heidelberg New York, 1977).

[31] K. S. Singwi, A. Sjölander, Phys. Rev. 120, 1093 (1960).

[32] V. G. Bhide, R. Sundaram, H. C. Bhasin, T. Bonchev, Phys. Rev. 3, 673 (1971).

[33] A. V. Zatovsky, Zh. Eksp. Teor. Fiz. 59, 500 (1970).

[34] A. Ya. Dzyublik, Ukr. Phys. J. 18, 1455 (1973).

[35] A. Ya. Dzyublik, Zh. Eksp. Teor. Fiz. 67, 1534 (1974).

[36] S. Chandrasekhar, Rev. Mod. Phys. 15, 1 (1943).

[37] G. A. Bykov, F. S. Hien, Zh. Eksp. Teor. Fiz. 43, 909 (1962).

[38] V. F. Sears, Can. J. Phys. 44, 1299 (1966); 45, 237 (1966). 


\section{ВИВЧЕННЯ РІЗНИХ ТИПІВ ДИФУЗІї В РІДИНАХ ЗА ДОПОМОГОЮ КВАЗІПРУЖНОГО РОЗСІЯННЯ НЕЙТРОНІВ}

О. Я. Дзюблик, В. І. Слісенко, О. А. Василькевич Інститут ядерних досліджень НАН Украйни просп. Науки, 47, Киӥв, 0зо28, Украӥна e-mail:dzyublik@kinr.kiev.ua

Виведено загальні рівняння для перерізу некогерентного квазіпружного розсіяння нейтронів рідиною 3 урахуванням неперервної транслящійної та обертальної дифузії так званих лагранжевих частинок (асощіяцій атомів чи молекул) та стрибків атомів водню в цих частинках. Розглянуто обидва типи неперервного руху на підставі рівнянь Ланжевена. Окрім того, проведено експерименти з розсіяння нейтронів на спиртах, результати яких добре узгоджуються з нашою теорією. 